\title{
Micronuclei induction in Rana catesbeiana tadpoles by the pyrethroid insecticide lambda-cyhalothrin
}

\author{
Marcela Alejandra Campana ${ }^{1}$, Ana María Panzeri ${ }^{1}$, Víctor Jorge Moreno ${ }^{2}$ and Fernando Noel Dulout ${ }^{3}$ \\ ${ }^{1}$ Departamento de Biología, Facultad de Ciencias Exactas y Naturales, Universidad Nacional de Mar del \\ Plata, Mar del Plata, Argentina. \\ ${ }^{2}$ Departamento de Ciencias Marinas, Facultad de Ciencias Exactas y Naturales, Universidad Nacional de \\ Mar del Plata, Mar del Plata, Argentina. \\ ${ }^{3}$ Centro de Investigaciones en Genética Básica y Aplicada (CIGEBA), Facultad de Ciencias Veterinarias, \\ Universidad Nacional de La Plata, La Plata Argentina.
}

\begin{abstract}
Pyrethroid lambda-cyhalothrin genotoxicity was evaluated using the micronucleus test in Rana catesbeiana tadpoles. The effects of concentration and exposure time on the micronuclei frequency were studied in blood smears obtained from tadpoles exposed to four concentrations $(0.02,0.1,0.2$ and $0.4 \mu \mathrm{g} / \mathrm{L})$ of the compound for $24,48,72$ and $96 \mathrm{~h}$ and 8, 15, 20 and 30 days. As a positive control, tadpoles were exposed to cyclophosphamide ( $5 \mathrm{mg} / \mathrm{L})$. The micronucleated cell frequency was expressed per 1,000 cells.

$R$. catesbeiana tadpoles exposed to increasing concentrations of lambda-cyhalothrin showed an increase in the micronuclei frequency in peripheral blood. Tadpoles exposed to cyclophosphamide (CP) also showed a significant increase in micronucleated erythrocytes which peaked after 15 days. These results suggest that $R$. catesbeiana tadpoles may provide a useful model for monitoring water pollution.
\end{abstract}

Key words: genotoxicity, micronucleus test, lambda-cyhalothrin, tadpoles.

Received: June 21, 2000; accepted: December 4, 2002.

\section{Introduction}

One of the main problems in the biomonitoring of genotoxic pollutants is the choice of test organism(s). Unequal sensitivity among species caused by different metabolic rates, physiological conditions and target organs can yield misleading results. For this reason, more than one species must be used to confirm the response to genotoxicants under experimental conditions.

The micronucleus test (MNT) developed by Schmid (1975) using mammalian bone marrow cells, has been applied extensively to test the genotoxicity of chemicals. The test has been successfully used with invertebrates, fish and amphibians, as a biological monitors of contaminated areas (in situ assay), and in the screening of compounds to determine their genotoxicity, after direct or indirect exposure in vivo (Jaylet et al., 1986; Hose et al., 1987; Brunetti et al., 1988; De Flora et al., 1993; Nepomuceno et al., 1997).

Send correspondence to Ana María Panzeri. Laboratorio de Genética, Departamento de Biología, Facultad de Ciencias Exactas y Naturales, Universidad Nacional de Mar del Plata, Funes 3250, 3er piso, (7600) Mar del Plata, Argentina. E-mail: apanzeri@mdp. edu.ar.
Micronuclei are formed by the loss of whole chromosomes or portions of chromosomes from daughter nuclei at mitosis and exist separately from the main nucleus of the cell. Micronuclei result either from chromosome breaks (clastogenic effects) or dysfunction of the spindle apparatus or centromere kinetochore complexes, with subsequent elimination of whole chromosomes (aneugenic effects). Compared to other cytogenetic assays, the several advantages in quantifying micronuclei include the speed and ease of analysis, and the lack of requirement for metaphase cells (Tucker and Preston, 1996)

Several authors have adapted the micronucleus test to assess the frequency of micronucleated cells in the fish $\mathrm{Um}$ bra pygmaea (Hooftman and de Raat, 1982), in amphibians such as Pleurodeles waltl, Ambystoma mexicanum and Xenopus laevis (Fernandez et al., 1993) and tadpoles of the anurans Rana catesbeiana and Caudiverbera caudiverbera (Krauter et al., 1987; Venegas et al., 1987).

In a recent paper we reported the dose-dependent micronuclei induction by pyrethroid lambda-cyhalothrin in the fish Cheirodon interruptus interruptus (Campana et al., 1999). The fish were treated with different doses of the pesticide for $24 \mathrm{~h}$ to 23 days. The micronuclei frequency in 
erythrocytes was highest after $24 \mathrm{~h}$ of treatment and decreased progressively thereafter. These results suggested that $C$. $i$. interruptus may be a suitable species for biomonitoring aquatic systems.

Fish generally show a higher acute toxicity to pyrethroids than frogs (Bradbury and Coats, 1989). Since current genotoxicity tests in amphibians are based on the observation of micronuclei in vivo (Jaylet et al., 1986; Gauthier et al., 1993; Ferrier et al., 1998), we examined the sensitivity of bullfrog (R. catesbeiana) tadpoles exposed to the same chemical as $C$. i. interruptus.

\section{Materials and Methods}

\section{Chemicals}

Lambda-cyhalothrin (trade name Karate) (Figure 1), CAS chemical name: $(R+S)$ - $\alpha$-cyano-3-(phenoxyphenyl) methyl - $(1 S+1 R)$ - cis - 3 - (z-2-chloro - 3,3,3, - trifluoroprop-1-enyl) - 2,2-dimethyl - cyclopropane-carboxylate, CASRN 91465-08-06, was obtained from Zeneca.

Cyclophosphamide (CP) (CAS No 50-18-0, Endoxan, Asta) was used as a positive control at a concentration of $5 \mathrm{ppm}(\mathrm{mg} / \mathrm{L})$. All test solutions were prepared immediately before each experiment.

\section{Animals}

Rana catesbeiana tadpoles were provided by Criadero de Ranas Chapadmalal (Chapadmalal Station, Batán, Argentina) and were acclimatized for 15 days prior to use. The larvae had an average weight and length of $3.44 \pm 1.06 \mathrm{~g}$ and $73.4 \pm 6.99 \mathrm{~mm}$, respectively, and were used at stage 28-29 which was characterized by rapid larva growth and development of the hind limbs (pro-premetamorphic larvae, based on the developmental table of Gosner (1960). This stage was selected for the micronucleus test because the larvae are large enough to allow cardiac puncture and provide a sufficient amount of blood for smears. In addition, this sampling interval corresponded to a period of intense hematopoiesis with active cell division in the circulating blood (Deparis, 1973). Red blood cells (RBCs) in lower vertebrates such as amphibians are nucleated and undergo cell division in the circulation, especially during the larval stages. These cells are therefore suitable for micronuclei detection which can be readily counted in blood smears.

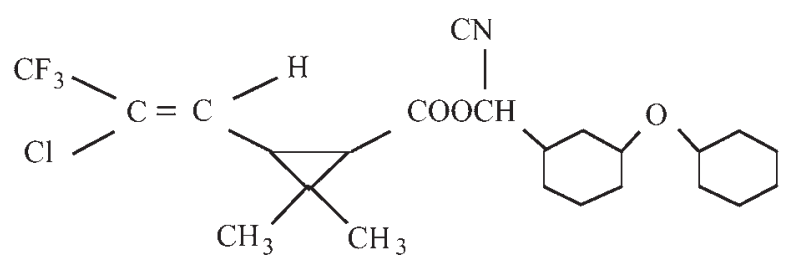

Figure 1 - Structure of the pyrethroid insecticide lambda-cyhalothrin $\left(\mathrm{C}_{23} \mathrm{H}_{19} \mathrm{ClF}_{3} \mathrm{NO}_{3}\right)$.

\section{Treatment and times for sample collection}

During the exposure period, the tadpoles were kept in $50 \mathrm{~L}$ aquaria, with aereated water at $21^{\circ} \mathrm{C}$. Preliminary experiments determined the concentration or maximum tolerated dose (MTD), at which the animals showed no reduction in survival and food uptake.

Four concentrations of lambda-cyhalothrin $(0.02,0.1$, 0.2 and $0.4 \mu \mathrm{g} / \mathrm{L}$ ) were tested. The water containing the compound and the food was changed every three days. A negative control and a positive control (using the well known mutagen cyclophosphamide, $5 \mathrm{mg} / \mathrm{L}$ ) were included. The micronuclei frequency in each group was scored after 24, 48, 72 and 96 hours, and 8, 15, 20 and 30 days of treatment.

\section{Blood smear preparation and analysis}

Tadpoles were anesthetized for approximately $2 \mathrm{~min}$ in a $5 \%$ solution of benzocaine and the blood samples were obtained by cardiac puncture, under a magnifying glass. Two peripheral blood smears for each animal were immediately prepared on clean slides, fixed in absolute methanol for $3 \mathrm{~min}$, and air dried. The following day, the slides were stained with $15 \%$ Giemsa solution for $5 \mathrm{~min}$. The micronuclei frequency was determined in 1,000 erythrocytes from each tadpole using 1,000x magnification. Coded and randomized slides were scored blind by a single observer. The frequency of micronucleated cells was expressed per 1,000 cells.

\section{Statistical analysis}

Where appropiate, the results were expressed as the mean \pm S.E.M. All data were tested for normality using the Wilks test. Since the data did not show a normal distribution, the statistical significance of the differences between treated and control tadpoles was evaluated using the non-parametric Kruskal-Wallis test. A value of $p<0.05$ was considered to indicate significance.

\section{Results}

The frequencies of micronuclei after treatment are shown in Table 1 and the time-response curves at each dose level are shown in Figure 2.

Mature $R$. catesbeiana erythrocytes are large, oval, nucleated cells (approximately $30 \mu \mathrm{m}$ long). The micronuclei observed showed the features described by Schmid (1975) (Figure 3).

The frequency of micronuclei increased with increasing concentrations of lambda-cyhalothrin (Figure 2).

There were no significant changes in micronuclei frequencies at the two lowest doses $(0.02$ and $0.1 \mu \mathrm{g} / \mathrm{L})$ of lambda-cyhalothrin. Tadpoles exposed to $0.2 \mu \mathrm{g} / \mathrm{L}$ showed a significant increase in micronuclei compared to the control group after $72 \mathrm{~h}(\mathrm{p}<0.05)$ and 15 days $(\mathrm{p}<0.01)$. 


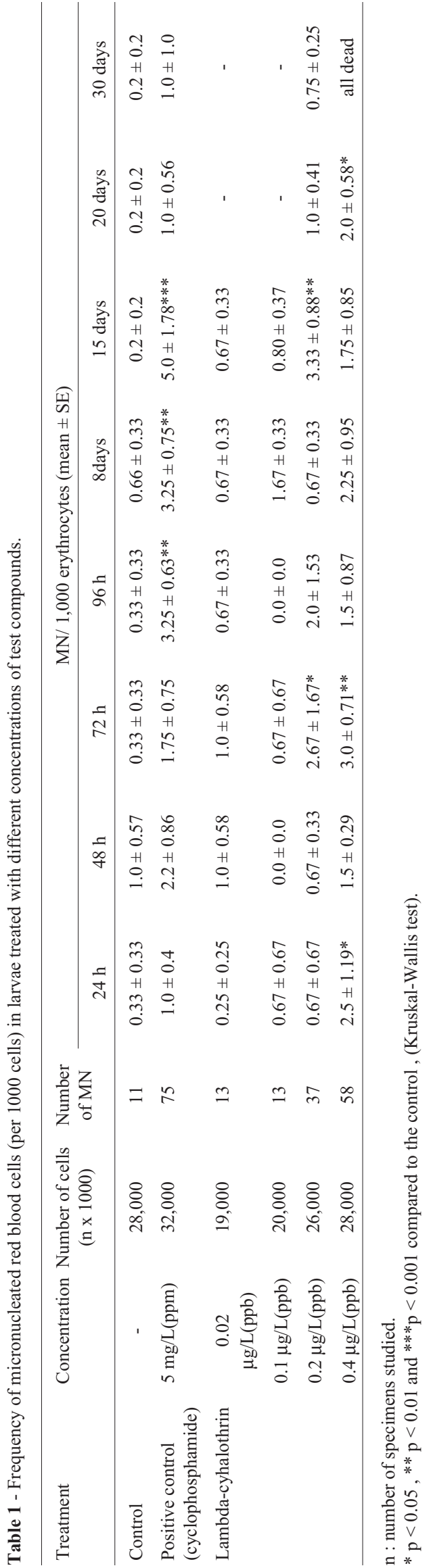

The highest concentration of the pyrethroid $(0.4 \mu \mathrm{g} / \mathrm{L})$ significantly increased the frequency of micronucleated erythrocytes compared to the negative control at 24, 48, and $72 \mathrm{~h}(\mathrm{p}<0.05, \mathrm{p} 0.01$, and $\mathrm{p}<0.01$, respectively), and at 20 days after exposure $(\mathrm{p}<0.05)$. Tadpoles exposed to this high dose of lambda-cyhalothrin did not survive more than 20 days of treatment.

Tadpoles exposed to cyclophosphamide (CP, positive control) showed a significant increase in micronucleated erythrocytes at $96 \mathrm{~h}(\mathrm{p}<0.01), 8$ days $(\mathrm{p}<0.01)$ and 15 days after exposure $(\mathrm{p}<0.0001)$. The micronuclei incidence increased gradually, reaching a highly significant peak at 15 days. After this lapse the frequency declined and was not different from the negative control (Figure 2).

Blood smears from tadpoles exposed to $\mathrm{CP}$ for 8 days or more showed a pronounced decrease in the number of $\mathrm{RBCs}$ and an increase in the destruction of erythrocytes
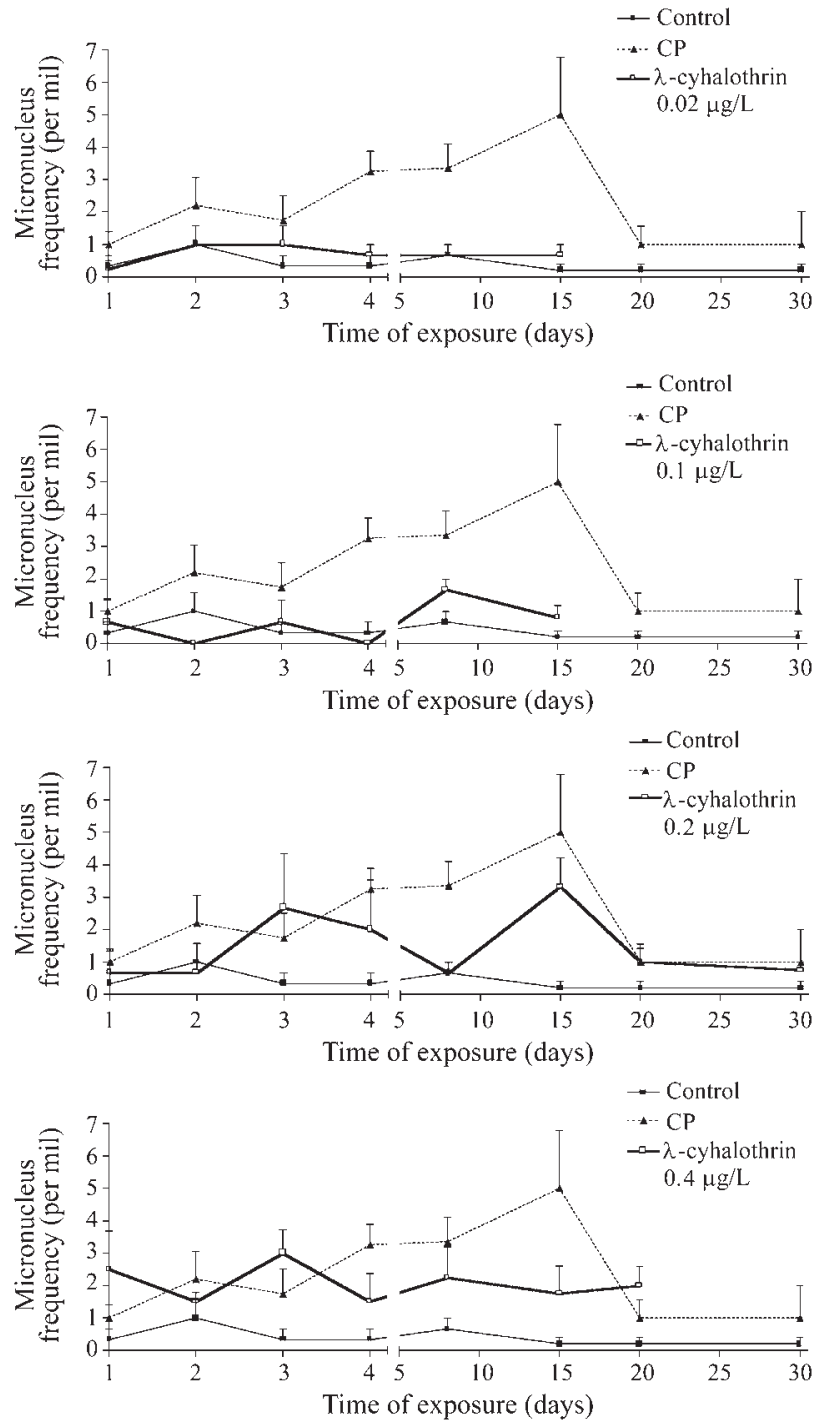

Figure 2 - Variation in the micronuclei frequency with time in each treated group. Each graph show control, CP (positive control) and the concentration of lambda-cyhalothrin tested. Data are the mean \pm S.E. 


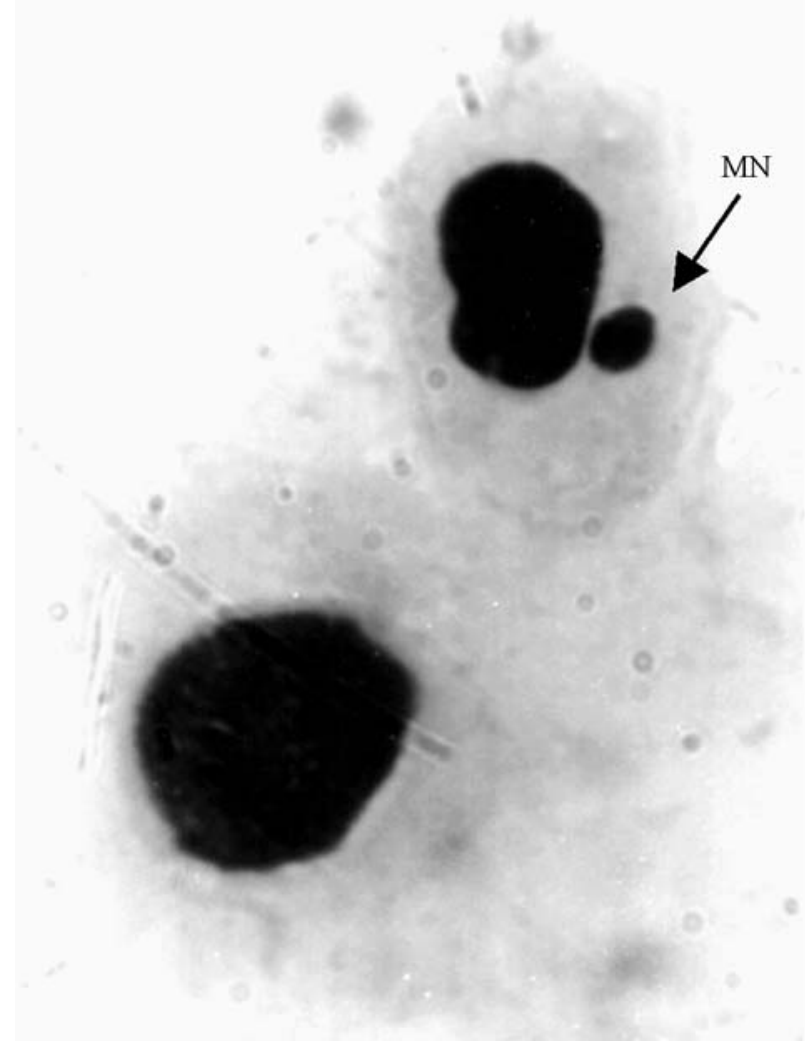

Figure 3 - Micronucleated erythrocyte (arrow) in R. Catesbeiana tadpole exposed to lambda-cyhalothrin. Giemsa-stained blood smear 1,000 x.

through fragmentation, collapse and rupture of the cell membrane. Precursor stages of mature erythrocytes were identified in the smears but few mature erythrocytes were observed.

\section{Discussion}

Siboulet et al. (1984) and Grinfeld et al. (1986) provided the first demonstration of the induction of micronuclei in RBCs of newt larvae (Pleurodeles waltl) exposed to X-rays and a variety of chemicals. Since then, 44 out of 78 chemical compounds tested in this model using the micronucleus test have been found to be genotoxic. In Xenopus, 9 out of 15 chemicals gave positive results with the micronucleus test (Ferrier et al., 1988). This test has also been used to examine the effects of $\gamma$-rays in $R$. catesbeiana (Krauter et al., 1987) and of chemicals in Caudiververa caudiververa (Venegas et al., 1987).

$\mathrm{CP}$ has been used as positive control in a variety of biological systems, with the highest micronuclei yield occurring after 15 days of treatment. These findings differ from the results obtained using the same concentration ( $5 \mathrm{ppm})$ in the fish C.i. interruptus, for which the highest micronuclei yield occurred after $24 \mathrm{~h}$. This finding may indicate that fish are more sensitive than amphibia. However, differences have also been reported between amphibian species. Thus, after treatment with 5 ppm CP the micronuclei was $87 \pm 10.6$ in Pleurodeles waltl (Fernandez et al., 1989), $3.5 \pm 0.4$ in Xenopus laevis (Fernandez et al., 1993), 1.7 in Rana temporaria and 1.05 in Xenopus laevis tadpoles (Rudek and Rozek, 1992). In addition, Van Hummelen et al. (1989), based on tests with ethyl methane sulfonate (EMS) and benzo(a)pyrene (BaP) in Xenopus laevis, reported a lower micronuclei frequency than that obtained by Jaylet et al. (1986) using Pleurodeles waltl.

The response of $R$. catesbeiana tadpoles to lambda cyhalothrin differed from that to $\mathrm{CP}$. Whereas the micronuclei frequency induced by $\mathrm{CP}$ increased progressively from $24 \mathrm{~h}$ to 15 days and decreased thereafter, with lambda-cyhalothrin there were differences between the responses to the doses. Thus, with a dose of $0.2 \mu \mathrm{g} / \mathrm{L}$, a bimodal response was found (Figure 2), with the highest micronuclei frequencies at $72 \mathrm{~h}$ and 15 days. With $0.4 \mu \mathrm{g} / \mathrm{L}$, the highest micronuclei yield appeared at $72 \mathrm{~h}$ and decreased slowly thereafter. The observation that with the lower doses $(0.02$ and $0.1 \mu \mathrm{g} / \mathrm{L})$ the micronuclei frequencies were similar to that of the negative control suggests a threshold effect of lambda cyhalothrin. As a result, multiple sampling times are needed to analyze the effects of different chemicals since the responses may vary according to the dose used.

Many amphibian populations are declining in number throughout the world (Bradford, 1991; Carey, 1993). In some cases, this phenomenon is associated with pollution by pesticides and heavy metals. Although environmental pollution may interfere with amphibian growth and development, the induction of genetic damage after chronic exposure to low doses of chemicals is perhaps the most important biological effect. The results obtained here with $R$. catesbeiana tadpoles exposed to CP and lambda-cyhalothrin indicate that this species provides a useful experimental model for monitoring water pollution.

\section{Acknowledgments}

This work is dedicated to the memory of Prof. Mónica López. The authors thank the Criadero de ranas Chapadmalal for supplying the tadpoles and Dr Martin Roubicek for helpful comments and suggestions, and for critical reading of the manuscript.

This work was supported in part by the Secretaría de Investigación y Desarrollo Tecnológico de la Universidad Nacional de Mar del Plata, Argentina (contract EXA 90/97).

\section{References}

Bradbury SP and Coats JR (1989) Comparative toxicology of the pyrethroid insecticides. Rev Environ Contam Toxicol 108:134-177.

Bradford DF (1991) Mass mortality and extinction in a highelevation population of Rana mucosa. J. Herpetol. 25:174177. 
Campana MA, Panzeri AM, Moreno VJ and Dulout FN (1999) Genotoxic evaluation of the pyrethroid lambda-cyhalothrin using the micronucleus test in erythrocytes of the fish Cheirodon interruptus interruptus. Mutat Res 438:155-161.

Carey C (1993) Hypothesis concerning the disappearance of boreal toads from the mountains of Colorado. Conserv Biol 7:355-362.

Deparis P (1973) Le sang circulant au cours de la croissance larvaire de Pleurodeles waltlii Michah (amphibien urodele). J Physiol 66:423-436.

Fernández M, Gauthier L and Jaylet A (1989). Use of the newt larvae for in vivo genotoxicity testing of water: results on 19 compounds evaluated by the micronucleus test. Mutagenesis $4: 17-26$.

Fernández M, LHaridon J, Gauthier L and Zoll-Moreux C (1993) Amphibian micronucleus test(s): a simple and reliable method for evaluating in vivo genotoxic effects of freshwater pollutants and radiations. Initial assessment. Mutat Res 292:83-99.

Ferrier V, Gauthier L, Zoll-Moreux C and LHaridon J (1998) Genotoxicity tests in amphibians: a review. Microscale Testing in Aquatic Toxicology: Advances, Techniques and Practice. CRC Press LLC, pp 507-519.

Gauthier L, Van der Gaag MA, L.Haridon J, Ferrier V and Fernandez M (1993) In vivo detection of waste water and industrial effluent genotoxicity: use of the newt micronucleus test (Jaylet test). Sci Total Environ 138:249-269.

Gosner KL (1960) A simplified table for staging anuran embryos and larvae with notes on identification. Herpetologica 16:183-190.
Grinfeld S. Jaylet A, Siboulet R, Deparis P and Chouroulinkow I (1986) Micronuclei in red blood cells of the newt Pleurodeles waltl after treatment with benzo(a)pyrene: dependence on dose, length of exposure, post-treatment time and uptake of the drug. Environ Mutagen 8:41-51.

Jaylet A, Deparis P, Ferrier V, Grinfeld S and Siboulet R (1986) A new micronucleus test using peripheral blood erythrocytes of the newt Pleurodeles waltl to detect mutagens in fresh-water pollution. Mutat Res 164:245-257.

Krauter PW, Anderson SL and Harrison FL (1987) Radiation-induced micronuclei in peripheral erythrocytes of Rana catesbeiana: an aquatic animal model for in vivo genotoxicity studies. Environ Mol Mutagen 10:285-296.

Rudek Z and Rozek M (1992) Induction of micronuclei in tadpoles of Rana temporaria and Xenopus laevis by the pyrethroid Fastac 10 EC. Mutat Res 298:25-29.

Schmid W (1975)The micronucleus test. Mutat Res 31:9-15.

Siboulet R, Grinfeld S, Deparis P and Jaylet A (1984) Micronuclei in red blood cells of the newt Pleurodeles waltlii Michah: induction with X-rays and chemicals. Mutat Res 125:275-281.

Van Hummelen P, Zoll C, Paulussen J, Kirsh-Volders M and Jaylet A (1989) The micronucleus test in Xenopus: a new and simple in vivo technique for detection of mutagens in fresh water. Mutagenesis 4:12-16.

Venegas W, Hermosilla I, Gavilan JF, Naveas R and Carrasco P (1987) Larval stages of the anuran amphibian Caudiverbera caudiverbera: a biological model for studies for genotoxic agents. Bol Soc Biol Conception 58:171-179.

Associate Editor: Yatiyo Yonenaga-Yassuda 